\title{
Optimal Pricing Strategy for Wireless Social Community Networks
}

\author{
Amin Mazloumian ${ }_{\dagger}$, Mohammad Hossein Manshaei ${ }_{\dagger}$, \\ Mark Félegyháził, and Jean-Pierre Hubaux $\uparrow$ \\ †Laboratory for Computer Communications and Applications, EPFL, Lausanne, Switzerland \\ \{seyyedamin.mazloumian,hossein.manshaei,jean-pierre.hubaux\}@epfl.ch \\ $\ddagger E E C S$, University of California at Berkeley \\ felegyha@eecs.berkeley.edu
}

\begin{abstract}
Wireless social community operators rely on subscribers who constitute a community of users. The pricing strategy of the provided wireless access is an open problem for this new generation of wireless access providers. In this paper, using both analytical and simulation approaches, we study the problem comprised of modeling user subscription and mobility behavior and of coverage evolution with the objective of finding optimal subscription fees. We compute optimal prices with both static and semi-dynamic pricing. Coping with an incomplete knowledge about users, we calculate the best static price and prove that optimal fair pricing is the optimal semidynamic pricing. Moreover, we have developed a simulator to verify optimal prices of social community operators with complete and incomplete knowledge. Our results show that the optimal fair pricing strategy significantly improves the cumulative payoff of social community operators.
\end{abstract}

\section{Categories and Subject Descriptors}

C.2.1 [ Network Architecture and Design]: Wireless communication; C.2.3 [Computer-Communication Networks]: Network Operations

\section{General Terms}

Design, Economics, Management

\section{Keywords}

Wireless Social Community, Pricing, Wireless Internet Service Providers

\section{INTRODUCTION}

Traditional licensed band wireless operators provide full coverage with high initial costs to construct the service infrastructure. The infrastructure is mainly comprised of base

Permission to make digital or hard copies of all or part of this work for personal or classroom use is granted without fee provided that copies are not made or distributed for profit or commercial advantage and that copies bear this notice and the full citation on the first page. To copy otherwise, to republish, to post on servers or to redistribute to lists, requires prior specific permission and/or a fee.

NetEcon'08, August 22, 2008, Seattle, Washington, USA

Copyright 2008 ACM 978-1-60558-179-8/08/08 ...\$5.00. stations and licensed spectrums. Wireless access providers that use WiMAX, EV-DO, or 3G technologies are instances of this type. On the contrary, wireless social community operators are set up at much lower costs and provide wireless coverage by the unlicensed band access points (APs) activated by subscribers who constitute a community of users. Presently, FON [2] is the largest wireless social community operator. In February 2006, FON announced that it had received $\$ 21.7$ million in equity financing from the Internet giants Google and Skype, and from the venture capital firms Index Venture and Sequoia Capital. They also made strategic agreements with the most popular ISPs, such as Cegetel in France with more than half million subscribers and BT in England with three millions users. This should enable them to increase their service coverage [1].

Relying on APs activated by users provides a viable alternative to the deployment of costly base stations and to the use of licensed spectrums for wireless communication. However, as the willingness of users to join such a community determines its evolution, there is no guarantee that the wireless social community network will reach full coverage. This willingness typically depends on various factors, such as the subscription fee and the mobility of users. In this paper, we model user subscription and mobility behaviors in different scenarios to assess the evolution of the wireless social community network. We compute optimal prices for a social community operator with static and semi-dynamic pricing. Assuming an incomplete knowledge about users, we show that fair pricing (when prices are proportional to the provided coverage) is an optimal strategy when the operator has to determine all its prices at the first instant (i.e., semi-dynamic pricing).

Furthermore, we have developed a simulator, called community simulator [3], to verify optimal prices of social community operators with complete and incomplete knowledge, and optimality of the fair pricing strategies. At the beginning of each simulation, operators of the simulator are capable of predicting the best static prices based on their knowledge about the mobile users and the city map. The simulator is described with the obtained numerical results and is publicly available [3]. To the best of our knowledge, this paper is the first to provide a detailed analysis of pricing strategies for this new type of wireless access providers.

In [4], Manshaei et al. investigate the competition between wireless social community and traditional licensed band operators with a game-theoretic approach. However, 
no mobility model is considered for users and the quality of service of wireless social community operator is proportional with the fraction of subscribed users with same value for all users. Whereas in our model, users choose destinations with different probability distributions and they explicitly assess the usefulness of the service by observing the wireless coverage provided by the social community and decide to subscribe based on their observations of coverage, e.g., a user with an AP near his home observes different wireless coverage from the user with no APs around his home. In our model, wireless social community operators may set lower prices for the users whose homes are near public places. Therefore, different prices may be adjusted for subscribers.

The paper is organized as follows. In Section 2, we explain our system model. In Sections 3 and 4, we discuss the pricing problem with static and fair pricing strategies. In Section 5, we present the community simulator in detail. In Section 6, we analyze numerical results and finally we conclude in Section 7.

\section{SYSTEM MODEL}

Our system model represents a service area in which a social community operator provides wireless access for a set of mobile users $N$ who intend to subscribe to the service. In our model decision times are instants of time at which the operator decides on prices of its service and users subscribe to or unsubscribe from the operator. We assume decision times are at the end of each month. Index $m$ designates the sequential order of decision times, i.e., $m \in\{0,1,2, \ldots\}$. Observation times are instants of time at which each user observes if he is provided with wireless access by the operator between decision times. There are $T_{o}$ observation times between each two decision time, i.e., $t \in\left\{1,2, \ldots, T_{o}\right\}$.

\subsection{System Graph}

We introduce a graph to model a city consisting of buildings, roads and APs. Buildings and road junctions are represented by graph vertices and road segments are represented by graph edges. Our definition of system graph should be rich enough to model the mobility of users and the topology of cities. The System graph, $M=(V, E)$, is a finite, planar, connected, weighted graph, where $V$ is the finite set of vertices (nodes) and $E$ is the finite set of bi-directional weighted edges. Nodes of the system graph are either road nodes or building nodes, as shown in Figure 1.

A node is a building node if its degree is equal to one. $B$ denotes the set of building nodes $B=\{v \in V \mid \operatorname{degree}(v)=$ $1\}$. A node is a road node if it is not a building node. $R$ denotes the set of road nodes $R=V \backslash B$. Building nodes are divided into three disjoint subsets: residential nodes $H$, workplaces $W$ and public places $J$. A residential node is a building node to which a user is assigned as its owner. The building node assigned to a user represents his home. $n_{h}$ denotes the home of user $n$. Workplace nodes $W$ represent workplaces of mobile users. There is a one-to-many mapping from the set of workplace nodes $W$ to the set of all mobile users $N . n_{w}$ denotes the workplace of user $n$. Places such as shopping centers and restaurants are represented by the set of public place nodes $J$. In order to simplify wireless coverage formulation, we introduce a binary function on pairs of nodes:

DeFinition 1. Let $d_{e}$ be the effective range of access points.

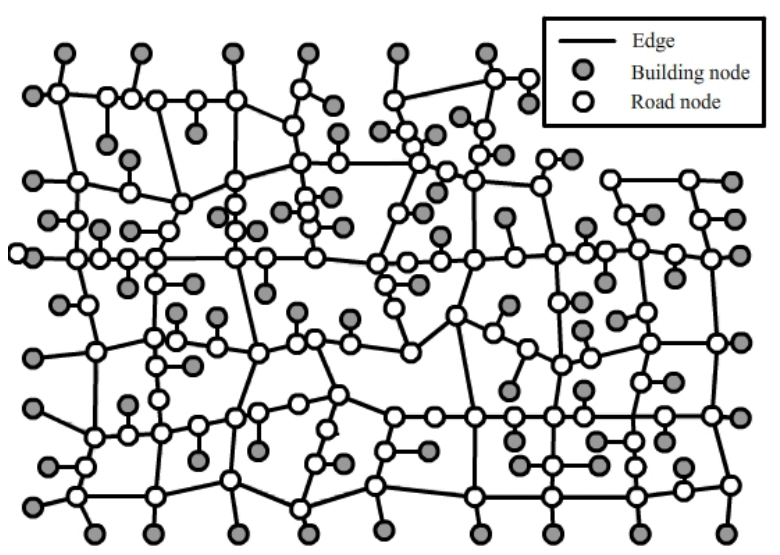

Figure 1: A System graph is a planar connected graph. The degree of building nodes is equal to one. The weights of edges are not shown in this figure.

The range function $d: V \times V \mapsto\{0,1\}$ is defined as:

$$
d\left(v, v^{\prime}\right)= \begin{cases}1 & \text { if }\left|v-v^{\prime}\right|<d_{e} \\ 0 & \text { otherwise }\end{cases}
$$

\subsection{Social Community Operator}

Each user activates an AP in his house when he joins the community by subscribing to the operator. In our model, the social community operator $\mathcal{S}$ is represented by $\mathcal{S}=$ $\left(N^{\mathcal{S}}, P, A P[0]\right)$, where $A P[0]$ is the initial set of nodes on which an AP belonging to $\mathcal{S}$ is active; $N^{\mathcal{S}}$ is the set of its subscribed users at decision times $N^{\mathcal{S}}=\left\{N^{\mathcal{S}}[1], N^{\mathcal{S}}[2], \ldots\right\}$, and $P$ is the set of its price sets for users at decision times $P=\{P[1], P[2], \ldots\}$, where $P[m]=\bigcup_{n \in N^{\mathcal{S}}[m]} P_{n}[m]$ and $P_{n}[m]$ is the price of operator $\mathcal{S}$ for user $n$ at decision time $m$. To specify our definition of social community operator we use the coverage function with the following definition:

Definition 2. Coverage function $C[m]$ for an operator at decision time $m$ is defined as:

$C(v, m)= \begin{cases}1 & \text { if it provides service on } v \text { at decision time } m \\ 0 & \text { otherwise }\end{cases}$

In our model, we assume all initial APs of social community operator $\mathcal{S}$ have been placed on public place nodes $J$ and workplace nodes $W$. Because these operators negotiate with owners and managers of public places and workplaces to make their service observable for potential mobile users. As the negotiation is not a part of our discussion, we assume that the initial access point locations $A P[0]$ of social community $\mathcal{S}$ are given at time 0 , and the operator never activates any other AP on public places or workplace nodes. In other words, $A P[0] \subseteq(J \cup W)$. We also assume that if all mobile users activate APs in their home nodes for the social community, there would be full coverage for all nodes of the system graph, i.e., $C(v, m)=1$. Note that, in some cities the community operators cannot provide full coverage even when all users subscribe to the service. Considering the above assumptions, the AP locations of any social community operator $\mathcal{S}$ are the residential nodes of subscribed mobile users or $A P[0]$. Hence, by using Definition 1, we can 
rewrite the coverage function presented in Definition 2 as:

$$
C(v, m)= \begin{cases}1 & \exists x \in\left(A P[0] \cup N^{\mathcal{S}}[m]\right) \mid d(x, v)=1 \\ 0 & \text { otherwise }\end{cases}
$$

The pricing strategy of an operator is static if it does not change its price set at decision times, i.e., $P[m]=P[1]$. The pricing strategy of an operator is semi-dynamic [5] if it determines its price set of all decision times at decision time $m=0$. The payoff of social community operator $\mathcal{S}$ for month $m$ is then:

$$
u[m]=\sum_{n \in N^{\mathcal{S}}[m]} P_{n}[m] .
$$

We can also calculate the cumulative payoff $U[m]$ of the operator at decision time $m$ by:

$$
U[m]=\sum_{i=1}^{m} u[i] .
$$

In our model, we say that the price sets $P$ and $P^{\prime}$ are equivalent, if and only if:

$$
\lim _{m \rightarrow \infty} \frac{U[m]}{U^{\prime}[m]}=1,
$$

where $U[m]$ and $U^{\prime}[m]$ are the cumulative payoffs of the operator with price sets $P$ and $P^{\prime}$, respectively.

\subsection{User Model}

Mobile users observe coverage provided by the operator at each observation time and at the following decision times they may subscribe or unsubscribe from the operator. In this section we explain different parameters of mobile users that determine their mobility and subscription behaviors.

\subsubsection{Mobility}

In our model, the mobile users move between nodes with a modified version of graph-based random waypoint algorithm [8], i.e., instead of choosing a random destination, mobile users choose their destination node with distribution $D^{c h}$. For any user $n$, destination choice distribution $D_{n}^{c h}$ indicates the probability that he chooses any building node $v \in B$ as his next destination. In other words, if he chooses destination at any observation time $t$, then $D_{n}^{c h}(v, t)$ is the probability that $n$ chooses $v$. We also assume that if mobile user $n$ chooses a public place node as his destination, his choice is uniform among the set of public place nodes $J$ :

$$
D_{n}^{c h}(j, t)=\frac{1}{|J|} \sum_{j^{\prime} \in J} D_{n}^{c h}\left(j^{\prime}, t\right) \quad \forall j \in J
$$

Similarly, if mobile user $n$ chooses a destination from other users' houses, his choice is uniform among the set of other users' houses:

$$
D_{n}^{c h}\left(h^{-n}, t\right)=\frac{1}{|H|-1} \sum_{h \in H^{-n}} D_{n}^{c h}(h, t) \quad \forall h^{-n} \in H^{-n}
$$

where $H^{-n}=H-\left\{n_{h}\right\}$. A shortest path algorithm (e.g. Dijkstra or Floyd-Warshal shortest path algorithms [9]) is used to find a path to the chosen destination. Before moving on the selected route, mobile users randomly choose a speed between $\left(\operatorname{speed}_{\min }, \operatorname{speed}_{\max }\right)$. Upon arrival, users stay at their destinations for an upper bounded random interval.
In our model, the mobility estimation of users is desirable for operators. For any user $n$, presence distribution $D_{n}^{x}$ indicates the probability that his location is node $v \in V$ at any observation time $t$, i.e.,

$$
D_{n}^{x}(v, t)=\operatorname{Pr}\{\text { location of } n=v \text {, at observation time } \mathrm{t}\} .
$$

The expected value of the number of observation times that the location of user $n$ is node $v$ would be:

$$
E_{D_{n}^{x}}(v)=\sum_{t=0}^{T_{o}} D_{n}^{x}(v, t),
$$

and the expected value of the number of observation times that his location is one of the nodes of set $L$ would be:

$$
E_{D_{n}^{x}}(L)=\sum_{v \in L} E_{D_{n}^{x}}(v)=\sum_{v \in L} \sum_{t=0}^{T_{o}} D_{n}^{x}(v, t) .
$$

Given $D_{n}^{c h}$, computing $D_{n}^{x}$ is not trivial. But, we can compute the expected value of the number of time steps a user spends in his home, workplace, public places and other users' houses, by simulation. Using Equation (4) and (5), we derive the expected value of the number of observation times that any user spends at public places and other users' houses.

$$
\begin{aligned}
& E_{D_{n}^{x}}(j)=\frac{1}{|J|} \sum_{j^{\prime} \in J} E_{D_{n}^{x}}\left(j^{\prime}\right) \quad \forall j \in J
\end{aligned}
$$

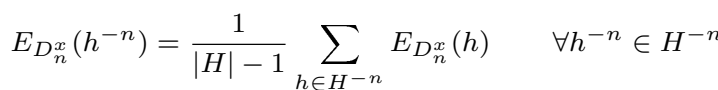

\subsubsection{Coverage Observation}

Mobile users observe the coverage provided by the operator expressed in Definition 2, at observation times with the frequency $f_{n}$. For instance, $f_{n}$ could be:

$$
f_{n}(v)= \begin{cases}5 & \text { for } v=n_{h} \\ 2 & \text { for }\left(v \in H \wedge v \neq n_{h}\right) \\ 20 & \text { for } v=n_{w} \\ 8 & \text { for } v \in J\end{cases}
$$

where user $n$ has his highest frequency of observations when he is at his workplace. Because, the service is more important for him at his workplace node $n_{w}$. The observed coverage $Q_{n}$ for user $n$ at decision time $m$ is defined as:

$$
Q_{n}[m]=\frac{\sum_{\forall n_{x}}\left(f_{n}\left(n_{x}\right) \times C\left(n_{x}, m\right)\right)}{\sum_{\forall n_{x}} f_{n}\left(n_{x}\right)},
$$

where $n_{x}$ is a traversed node between decision times $m$ and $m-1$. A node may be traversed and counted several times.

\subsubsection{Subscription and payoff function}

Any mobile user $n$ subscribes to the operator if his payoff is positive. In our model, the Payoff $u_{n}[m]$ of user $n$ at decision time $m$ is defined as:

$$
u_{n}[m]=a_{n} \times Q_{n}[m-1]-P_{n}[m],
$$

where parameter $a_{n}$ indicates the importance of provided coverage $Q_{n}$ for user $n$. $a_{n}$ has distribution $D^{a_{n}}$ over all users. At each decision time, any mobile user subscribes or stays subscribed to the operator if his payoff is positive. If a subscribed mobile user's payoff becomes negative, he will unsubscribe from the operator with no penalty.

Note that we assume operators know system graph $M$, probability distribution of parameter $a_{n}$ and the expected number of observation times users spend in their workplaces, 
other users' houses, road nodes, public place nodes and at home. Moreover, operators know the frequency of observations $f_{n}$, and subscriptions/unsubscriptions of users at previous decision times.

\section{STATIC PRICING STRATEGY}

Having defined the system model, we can now find an optimal price set for the operators with complete knowledge in different scenarios. The aim of a social community operator with static pricing strategy is to find the optimal price set that maximizes its cumulative payoff. In order to achieve this goal, the operator predicts its cumulative payoff for different price sets and chooses the optimal one.

\subsection{Pricing with Complete Knowledge}

A social community operator with complete knowledge not only knows the distribution of parameter $a_{n}$ over all users but also knows the exact value of parameter $a_{n}$ of each user. We explain how the operator computes its cumulative payoff for a price set $P$. The operator calculates its coverage at decision time 0 from its initial access point locations $A P[0]$. With complete knowledge, it determines whether or not each user subscribes to or unsubscribes from it. Therefore, it finds locations of its access points $A P[1]$ at decision time 1 . By repeating this procedure, the operator can calculate its cumulative payoff at any decision time. Here we describe the computation of coverage $Q_{n}$ for any user $n$ based on AP locations at decision time $m$. By knowing system graph $M$ and locations of access points $A P[m]$, operator $\mathcal{S}$ calculates coverage function $C$ from which it finds the observed coverage of each user:

$$
\begin{aligned}
Q_{n}[m] & =\frac{E_{D_{n}^{x}}\left(f_{n} \times C\right)}{E_{D_{n}^{x}}\left(f_{n}\right)} \\
& =\frac{\sum_{v \in V} \sum_{t=0}^{T_{o}}\left(D_{n}^{x}(v, t) \times f_{n}(v) \times C(v, m)\right)}{\sum_{v \in V} \sum_{t=0}^{T_{o}}\left(D_{n}^{x}(v, t) \times f_{n}(v)\right)} \\
& =\frac{\sum_{v \in V}\left(f_{n}(v) \times C(v, m) \times E_{D_{n}^{x}}(v)\right)}{\sum_{v \in V}\left(f_{n}(v) \times E_{D_{n}^{x}}(v)\right)}
\end{aligned}
$$

Using Equations (8) and (9), we can rewrite (12) as:

$$
\begin{aligned}
Q_{n}[m] & =\frac{f_{n}\left(n_{h}\right) \times C\left(n_{h}, m\right) \times E_{D_{n}^{x}}\left(n_{h}\right)}{\sum_{v \in V}\left(f_{n}(v) \times E_{D_{n}^{x}}(v)\right)} \\
& +\frac{f_{n}\left(n_{w}\right) \times C\left(n_{w}, m\right) \times E_{D_{n}^{x}}\left(n_{w}\right)}{\sum_{v \in V}\left(f_{n}(v) \times E_{D_{n}^{x}}(v)\right)} \\
+ & \frac{E_{D_{n}^{x}}(J) \times \sum_{v \in J}\left(f_{n}(v) \times C(v, m)\right)}{|J| \times \sum_{v \in V}\left(f_{n}(v) \times E_{D_{n}^{x}}(v)\right)} \\
+ & \frac{E_{D_{n}^{x}}\left(H^{-n}\right) \times \sum_{v \in H^{-n}}\left(f_{n}(v) \times C(v, m)\right)}{(|H|-1) \times \sum_{v \in V}\left(f_{n}(v) \times E_{D_{n}^{x}}(v)\right)} \\
+ & \frac{E_{D_{n}^{x}}(v) \times \sum_{v \in R}\left(f_{n}(v) \times C(v, m)\right)}{\sum_{v \in V}\left(f_{n}(v) \times E_{D_{n}^{x}}(v)\right)}
\end{aligned}
$$

The computation of all terms of Equation (13) is trivial, except the last term. The point is that the social community operator cannot simply assume that mobile users uniformly spend their time on road nodes:

$$
E_{D_{n}^{x}}(r) \not \frac{E_{D_{v}^{n}}(R)}{|R|} \quad \forall r \in R .
$$

As we are using a shortest path algorithm, we give more weight to those nodes that appear more often on shortest paths between building nodes. Let $T(v)$ be the set of all shortest paths between building nodes that go through node $v$. Each shortest path goes through each node at most once (There is no cycle in a shortest path). With this approximation we obtain:

$$
E_{D_{n}^{x}}(r) \simeq \frac{E_{D_{n}^{x}}(R) \times|T(r)|}{\sum_{v \in R} T(v)} .
$$

Having calculated the observed coverage by users $Q_{n}$, the operator can compute its payoff at all decision times for a given price set. By changing the initial price set, it can find the optimal price set that maximizes its cumulative payoff.

\subsection{Pricing with Incomplete Knowledge}

A social community operator with incomplete knowledge does not know the exact value of the preference parameter $a_{n}$. The operator can predict the future of the system based on the distribution of the parameter $D^{a_{n}}$. Instead of using coverage function $C$ to calculate observed coverage $Q_{n}$, the operator uses the expected value of coverage function $E(C)$ :

$$
\begin{aligned}
E(C(v, m)) & =\operatorname{Pr}\{C(v, m)=1\} \\
& =\operatorname{Pr}\left\{\bigcup_{v^{\prime} \mid d\left(v^{\prime}, v\right)=1}\left(v^{\prime} \in A P[m]\right)\right\} \\
& =1-\operatorname{Pr}\left\{\bigcap_{v^{\prime} \mid d\left(v^{\prime}, v\right)=1}\left(v^{\prime} \notin A P[m]\right)\right\} \\
& =1-\prod_{v^{\prime} \mid d\left(v^{\prime}, v\right)=1} \operatorname{Pr}\left\{v^{\prime} \notin A P[m]\right\}
\end{aligned}
$$

There are two different cases for the probability that an AP is not located on node $v^{\prime}$. Any access point node in $A P[m]$ is a public place or workplace node or a mobile user's home node. If it is a public place node or a workplace node, it is initially defined 0 or 1 in the initial coverage of the operator, but if it is a residential node and user $n$ is its owner at decision time $m>0$ :

$$
\begin{aligned}
\operatorname{Pr}\left\{v^{\prime} \notin A P[m]\right\} & =\operatorname{Pr}\left\{n \notin N^{\mathcal{S}}[m]\right\} \\
& =\operatorname{Pr}\left\{a_{n} \times Q_{n}[m-1]-P_{n} \leq 0\right\} \\
& =\operatorname{Pr}\left\{a_{n} \leq \frac{P_{n}}{Q_{n}[m-1]}\right\} \\
& =\int_{0}^{\frac{P_{n}}{Q_{n}[m-1]}} D^{a_{n}}(\mathrm{x}) \mathrm{dx}
\end{aligned}
$$

and the cumulative payoff at decision time $m$ would be:

$$
\begin{aligned}
U[m] & =\sum_{k=1}^{m} u[k] \\
& =\sum_{k=1}^{m} \sum_{n \in N^{\mathcal{S}}[k]} P_{n} \times \operatorname{Pr}\left\{n \in N^{\mathcal{S}}[k]\right\} \\
& =\sum_{k=1}^{m} \sum_{n \in N^{\mathcal{S}}[k]} P_{n} \times \operatorname{Pr}\left\{a_{n}>\frac{P_{n}}{Q_{n}[k-1]}\right\} \\
& =\sum_{k=1}^{m} \sum_{n \in N^{\mathcal{S}}[k]} P_{n} \times \int_{\frac{P_{n}}{Q_{n}[k-1]}}^{\infty} D^{a_{n}}(\mathrm{x}) \mathrm{dx}
\end{aligned}
$$


The operator evaluates different price sets to find the price set that maximizes its cumulative payoff. We have compared predicted payoffs by simulation for different prices when the operator sets the same price for all users, in Section 6 .

\section{FAIR PRICING STRATEGY}

In this section, we assess semi-dynamic pricing strategies of operators. First, we define fair pricing:

Definition 3. A pricing strategy of an operator is fair if for any user $n$, its price at decision time $m-1$ is a constant factor $c_{n}$ of its predicted coverage for the user at month $m-$ 1: $P_{n}[m]=c_{n} \times Q_{n}[m-1]$, where fairness constant $c_{n}$ is defined at decision time 0 , and $Q_{n}[m-1]$ is the predicted value of provided coverage for decision time $m-1$, computed at decision time 0.

As the price set is determined at decision time $m=0$, fair pricing strategies are semi-dynamic [5]. We prove that for each semi-dynamic pricing of an operator, there exists an equivalent fair pricing if there exists a decision time after which its user set and price set do not change. Note that there are cases where even if the operator does not change its price set, its set of subscribed users changes forever. Hence, the assumption about the existence of such a decision time is necessary. First, we find membership probabilities of users.

Lemma 1. The probability that any mobile user $n$ subscribes or stays subscribed to a social community operator (with fair pricing) at decision time $m>0$ with fairness constant $c_{n}$ is $\int_{c_{n}}^{\infty} D^{a_{n}}(x) \mathrm{d} x$.

THEOREM 1. For each semi-dynamic pricing of a social community operator, there exists an equivalent fair pricing if there exists a decision time after which its user set and price set do not change.

Proof. The proofs are given in [6].

\subsection{Optimal Fair Prices}

The goal of any operator is to find the best fairness constants $c_{n}$ that maximizes its payoff. The expected value of coverage on any node $v$ is calculated in Equation (14). If node $v$ is a public place node or a workplace node, $\operatorname{Pr}\left\{v^{\prime} \notin\right.$ $\left.A P^{i}[\mathrm{~m}]\right\}$ is initially defined 0 or 1 in initial coverage of the operator. Otherwise for any residential node $v^{\prime}$ with owner $n$, we can use Lemma 1 to calculate the probability:

$$
\begin{aligned}
\operatorname{Pr}\left\{v^{\prime} \notin A P[m]\right\} & =1-\operatorname{Pr}\left\{n \in N^{\mathcal{S}}[m]\right\} \\
& =\int_{0}^{c_{n}} D^{a_{n}}(x) \mathrm{d} x
\end{aligned}
$$

Then the payoff of the operator at month $m$ is:

$$
\begin{aligned}
u[m] & =\sum_{n \in N}\left(P_{n} \times \operatorname{Pr}\left\{n \in N^{\mathcal{S}}[m]\right\}\right) \\
& =\sum_{n \in N}\left(c_{n} Q_{n} \operatorname{Pr}\left\{n \in N^{\mathcal{S}}[m]\right\}\right) \\
& =\sum_{n \in N}\left(c_{n} Q_{n} \int_{c_{n}}^{\infty} D^{a_{n}}(x) \mathrm{d} x\right),
\end{aligned}
$$

where the provided coverage for any user $n$ can be computed as a linear function of these membership probabilities:

$$
Q_{n}[m]=\frac{\sum_{v \in V} E_{D_{n}^{x}}(v) \times f_{n}(v) \times E(C(v, m))}{E_{D_{n}^{x}}\left(f_{n}\right)} .
$$

Notice that all parameters of payoff $u[m]$ do not change after decision time 1 . Therefore, the maximum payoff is:

$$
u^{o p t}[m]=u^{o p t}[2]=\max _{\left\{c_{1}, \ldots, c_{|N|}\right\}}(u[2]) .
$$

With the payoff function and its derivative with all fairness constants, the optimization can be done with any nonlinear optimization method such as Levenberg-Marquardt [7] or gradient descent. Note that the above calculated optimal fair pricing strategy assigns different prices according to the locations of users' home nodes and provided coverage.

\section{COMMUNITY SIMULATOR}

Our developed community simulator, with high flexibility and good performance, simulates all possible scenarios of our model with high precision [3]. The simulator supports any number of operators with any pricing strategy. Before beginning a simulation, community simulator generates a city map with an open source random map generator [3]. The following parameters can be modified in the community simulator: the size of the city, road density, adjacency distances of road nodes, map uniformity, road smoothness and building density. The parameters of the map generator are set to generate a $4 \mathrm{~km} \times 4 \mathrm{~km}$ map. Community simulator works with real time format $y y / m m / d d . h h: m m: s s$. The format was preferred to the cycle based timer, because mobile users choose their destination according to the time of day. Every 15 simulated seconds, all mobile users observe coverage. Mobile users reset all their observations at the end of each month.

Choice-destination distribution is defined in a way that results in the expected value of the number of observation times that any user $n$ is present at a set of nodes $L$ as:

$$
E_{D_{n}^{x}}(L)= \begin{cases}48-50 \% & \text { for } L=\left\{n_{h}\right\} \\ 0-1 \% & \text { for } L=H-\left\{n_{h}\right\} \\ 9-12 \% & \text { for } L=R \\ 29-31 \% & \text { for } L=\left\{n_{w}\right\} \\ 9-11 \% & \text { for } L=J\end{cases}
$$

Before the simulator timer starts, we calculate all-pair shortest paths with the Floyd-Warshal algorithm [9]. Therefore, there is no need to run a shortest path routing during simulation. Mobile users choose their speed for each destination randomly between $10 \mathrm{~km} / \mathrm{h}$ and $30 \mathrm{~km} / \mathrm{h}$. After reaching their destinations, they stay from 1 to 3 hours at their destination nodes and then they choose the next destination and repeat the steps again.

\section{NUMERICAL RESULTS}

In our simulation, we generate the downtown of a densely populated city with 1104 buildings, 884 residential nodes and 2048 road nodes. A social community operator with an initial coverage of $10 \%$ of public place and workplace nodes (with an equal number of initially covered workplace and public place nodes) provides coverage in the simulated city. The distribution of parameter $a_{n}$ is defined to be uniform 


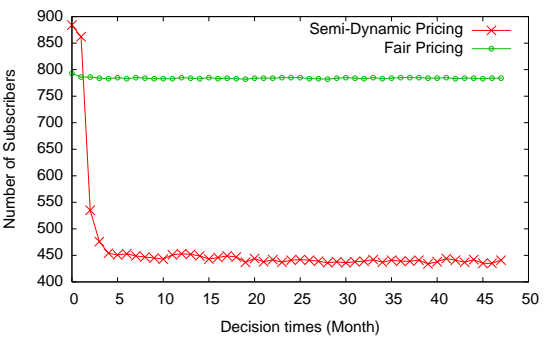

(a)

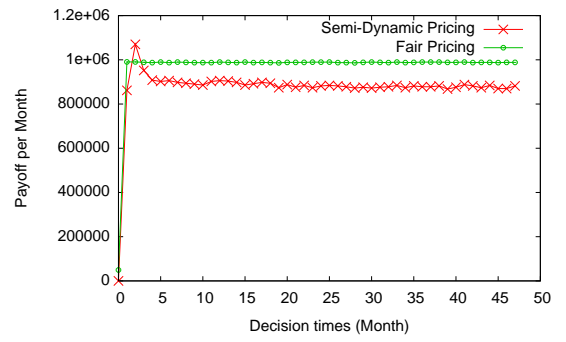

(b)

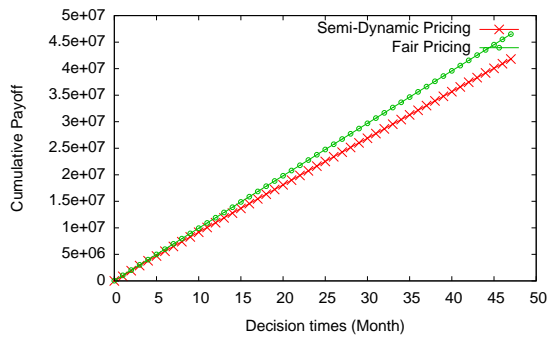

(c)

Figure 3: Performance analysis of wireless social community operator with our developed community simulator. Fair pricing and a semi-dynamic pricing $\left(P[0]=0, P[1]=1000, P\left[2^{+}\right]=2000\right)$ have been used for four years (48 months): (a) Number of subscribed users per month, (b) Monthly payoff of social community operator, (c) Cumulative payoff of operator.

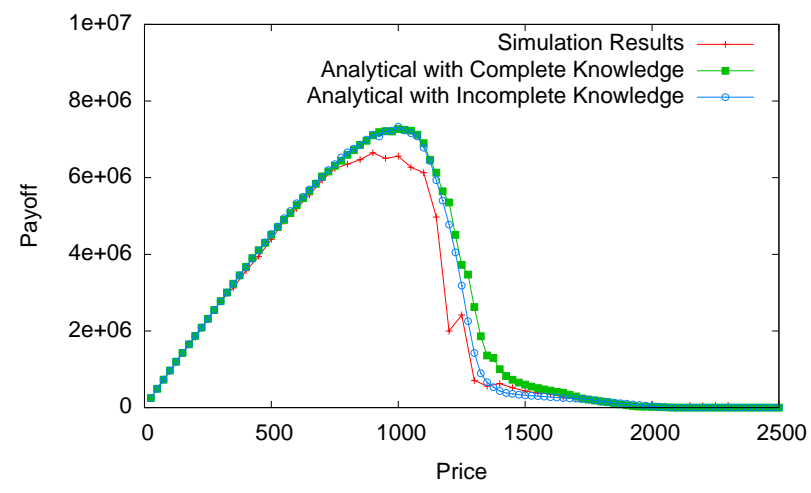

Figure 2: Predicted payoff with complete and incomplete knowledge and the occurred cumulative payoff of a social community operator in 12 months.

between 1000 and 4000, over all users. The implemented social community operator can predict its payoff for different static prices with complete or incomplete knowledge at the first instance of simulation. In Figure 2, we compared predicted cumulative payoff of the operator of 12 months with its occurred payoff. The results show that with complete and incomplete knowledge, the optimal static price 1000 is predicted and is equal to the optimal occurred payoff. As we have proved in Section 4, optimal fair pricing is the optimal semi-dynamic pricing of a social community operator. In our simulations, we found optimal fairness constants of users by applying gradient descent. We also considered another semi-dynamic pricing with prices 0 and 1000 for the first and second months and 2000 for all the following months. With price 0 (free service), all users subscribe and the provided coverage would highly increase. The improved coverage helps to keep more users, even with the high prices of the following months. Figure 3 shows the monthly payoff, the cumulative payoff, and the number of subscribed users with these two pricing strategies during 4 years. We observe that the proposed optimal fair pricing strategy outperforms the considered semi-dynamic pricing strategy and improves the cumulative payoff about $10 \%$. Moreover, the number of subscribed users and the monthly payoff are more stable using the proposed optimal fair pricing strategy.

\section{CONCLUSIONS}

We have computed optimal strategies in different scenarios of wireless social community operators with complete and incomplete knowledge. The required information of the proposed model can be gathered from traditional statistical studies (e.g. subscription forms) and/or data mining. To gain complete knowledge, operators need to distinguish personal subscription preferences. We have shown that social community operators should set different subscription fees for users to maximize their payoffs. The reason is that mobile users observe different wireless coverage based on their preferred locations. Furthermore, lower subscription fees should be adjusted for owners of houses (potential AP locations) near public places or workplaces. In terms of future work, contract-based subscriptions and throughput of the network could also be considered. Moreover, simulations can be done on maps of real cities with statistical data as input.

\section{REFERENCES}

[1] Fon friend. http://www.fon.com/en/info/fonFriends.

[2] Fon wireless access provider. http://www.fon.com.

[3] EPFL-IC-LCA1. Wireless social community simulator. http://wiki.epfl.ch/wsc.

[4] M. H. Manshaei, J. Freudiger, M. Felegyhazi, P. Marbach, and J.-P. Hubaux. On Wireless Social Community Networks. In IEEE INFOCOM, 2008.

[5] S. Markovitch and Y. Sella. Learning of resource allocation strategies for game playing. Computational Intelligence, 12(1):88-105, 1996.

[6] A. Mazloumian, M. H. Manshaei, M. Felegyhazi, and J.-P. Hubaux. Optimal pricing strategy for wireless social community networks. EPFL Research Report, (LCA-REPORT-2008-013), 2008.

[7] W.H. Press et al. Numerical recipes in C. Cambridge University Press Cambridge, 1992.

[8] J. Tian, J. Hähner, C. Becker, I. Stepanov, and K. Rothermel. Graph-based mobility model for mobile ad hoc network simulation. In Annual Simulation Symposium, 2002.

[9] Douglas B. West. An Introduction to Graph Theory. Prentice Hall, 1996. 